\title{
A remote lecturing system offering complementary nonverbal information in lectures
}

\begin{abstract}
This paper proposes a remote lecturing system, which has some features to complement nonverbal information in lectures. The proposed system provides students entire sight of the teacher, the blackboard, lecture slides, and so on with low definition video. The students can get the high definition pictures of where they would like to watch by clipping a region on the video. They also can write notes directly on the clipped pictures. The system collects data of clipped regions and wrote notes from the students, and offers its users the data to complement nonverbal information in the lecture. The system also provides the teacher and the students the video of others' faces depending on the situations of the lecture. This paper also organises the verbal and nonverbal information in several situations in a lecture.
\end{abstract}

Keywords: information in a lecture, remote lecture, verbal information, nonverbal information

Reference 


\section{Introduction}

Distance learning with the Internet has rapidly spread over the world. In the United States, over 6.1 million students enrolled on at least one online course during the fall semester in 2010 [2]. Many universities in other countries also offer online courses to their students $[3,4,8,10]$. There are many inter-university consortia, and the participating universities of a consortium are sharing their lectures and learning resources each other [4, 5, 9]. The universities, however, are often distant from the others; thus, they offer the others online lectures and learning resources.

On the other hand, the importance of lifelong learning is also increasing in many countries. For example, the council of the European Union states a benchmark in 2009[11]:

By 2020, an average of at least $15 \%$ of adults should participate in lifelong learning.

Some of lifelong learners participate in lectures at universities; however, they are often distant from universities in which they would like to enroll. Thus, they also need to participate in lectures online.

There are synchronous and asynchronous online lectures from the temporal viewpoint, and this study only focuses on synchronous online lectures. The teacher of a lecture typically has face-to-face (on-site) students in a classroom, and the online students watch video distributed from the classroom at designated room for online lectures, their home, or other places. The designated room is equipped with enough wide network connections and large screens. On the other hand, there are a narrow network connection and a small display at their home and other places.

This paper describes a remote lecturing system to offer remote participants at home or other places. Moreover, we examine verbal and nonverbal information in a lecture to consider the system functionalities for providing supplemental information.

\section{Style of Lecture Video}

We consider the styles of lectures as follows:

- a teacher talks to students, and the teacher / the student sometimes make questions and answers

- students discuss, collaborate, or cooperate in their group

In the former type lectures, the teacher one-sidedly talks to the students and writes and shows complementary information including figures, equations, etc. on the blackboard and the slides. On the other hand, the students participating the lecture actively talk and collaboratively work with other students, and the teacher mainly manages and facilitates the students in the latter type lectures. In this paper, we focus on the former type lectures as the first step of this work because these are the simplest form of lectures.

Remote lectures are separated into two categories: asynchronous and synchronous. Asynchronous courses typically offer students a recorded lecture and slides or webbased learning materials stored on a learning management system. Synchronous courses 
representatively distribute a lecture video and slides from a lecture room to remote lecture rooms or students' computers. Previously, the lecture video was typically low definition, less than or equals to $640 \times 480$, and was not fit to take entire picture of a blackboard, a lecturer, and a projected slide to the video. Abler et al. pointed out as follows [1]:

Common videoconference and distance learning systems provide students with a traditional television-like view of the instructor and/or the instructor's materials. This requires the instructor to use specially prepared materials to effectively reach the remote students.

There are various online participants, and universities need to open various lectures for them. However, it is difficult to revise existent lectures to fit the above-mentioned requirement. We consider, therefore, a remote lecturing system, which distributes high definition lecture video, not a traditional television-like view, but including entire the blackboard, a lecturer, and the slides. Such video includes entire the students' sight, and does not need specially prepared materials.

Kunimune et al. prepared two types of high definition lecture videos, one of them takes entire picture of the blackboard and a lecturer and its camera angle is fixed, and another one takes limited scope and its camera angle moves to the place where teacher is explaining. They projected video of each type on a screen in approximately real size, and students took lectures with each type video. According to the experimental result, the former one is better than the latter one about realistic sensation, concentration, note taking, and fatigue [7]. The characters on the blackboard written by the teacher are enough observable because the video was recorded in high definition and projected in approximately real size. Some of the students answered a questionnaire that the video in fixed camera angle is better because they can watch anywhere in the blackboard. The students sometimes need to watch somewhere, where the teacher has already explained, to take notes or refer the former explanations.

Lecture rooms designed for remote lectures offer students high definition video on large screens. In this case, the students have to travel to the designated room, and the universities offering remote lectures need to prepare many rooms. On the other hand, the students can also participate in remote lectures with their computers at their home or some other places. There are some problems in this case: display size is too small to recognise the writing on the blackboard and the slides in a high definition lecture video, and network bandwidth is not enough to receive a high definition lecture video.

Figures 1(a), 1(b), and 1(c) show three pictures clipped from an actual lecture video in different definitions. There are the large characters are on the left side of the blackboard, and the diagrams and the small characters are on the right side. In the video shown in Fig. 1(a), the some characters on the left side can be recognised; however some complicated characters (Japanese Kanji characters) on the left side and the diagrams and small characters on the right side are difficult to be read. In the video shown in Fig. 1(b), the diagrams and the small characters on the right side are also difficult to be recognised. In the high definition video shown in Fig. 1(c), almost all of characters and diagrams on the blackboard can be recognised with large displays; however, it is difficult to recognise the small characters in the diagrams when the video is played on small displays. 
Figure 1 An example of differences among video definitions.

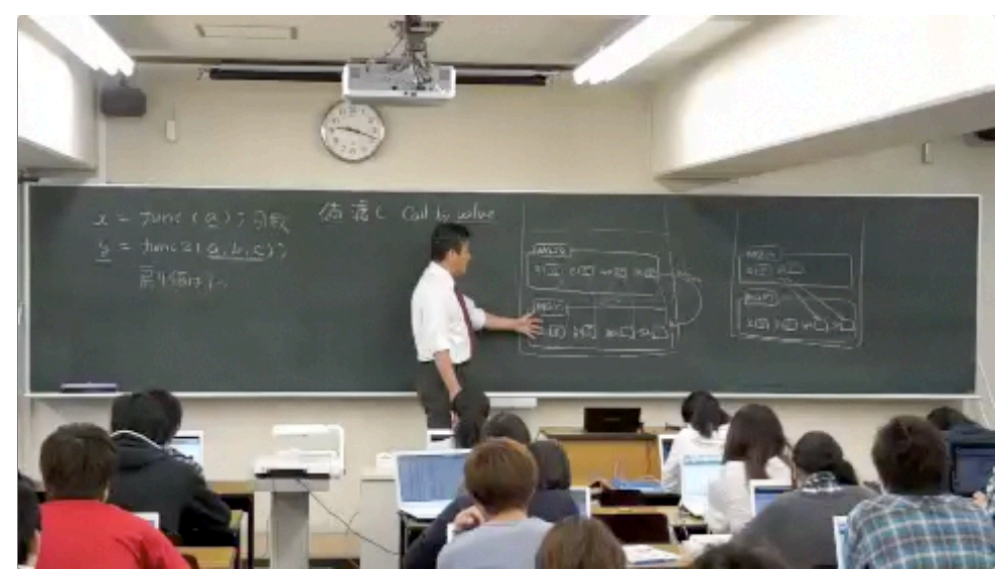

(a) Low definition video $(480 \times 272$ pixels, $1 \mathrm{Mbps})$.

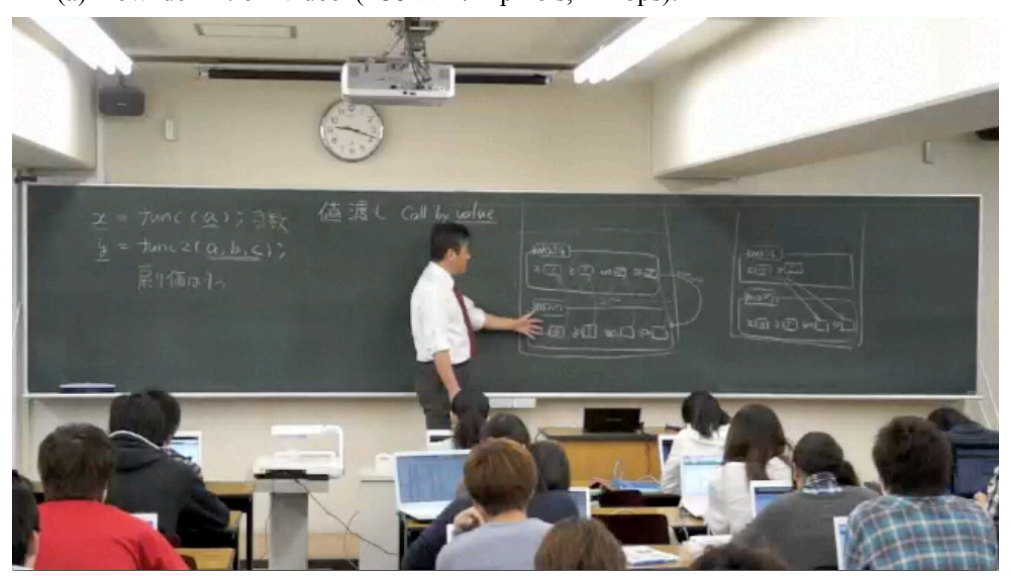

(b) Medium definition video $(640 \times 360$ pixels, $1.7 \mathrm{Mbps})$.

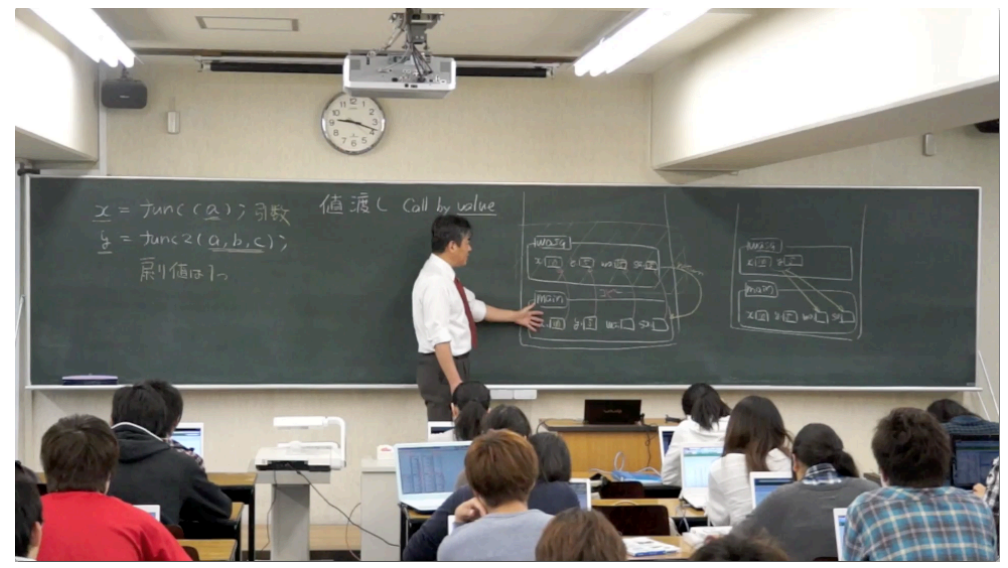

(c) High definition video $(1920 \times 1080$ pixels, $20 \mathrm{Mbps})$. 


\section{Information in a Lecture}

\subsection{Verbal and Nonverbal Information}

There are verbal and nonverbal information in the lectures. Verbal information includes the utterances and the writing on the blackboard and the slides. Nonverbal information includes the pitch of voices, movements (gazes, facial expressions, gestures, and activities), and so on.

Video with audio can include almost all of verbal information, and it also contains a part of nonverbal information such as facial expressions, gestures, and so on. However, it is difficult to include some kinds of nonverbal information. For example, the gaze directions of both the teacher and the students are lost by the "Mona Lisa effect." Hirata et al. explain the "Mona Lisa effect" as follows [6]:

The Mona Lisa effect is a cognitive phenomenon as follows: when a viewer looks at a person displayed on a screen looking at the front, the viewer feels that the person always appears to follow the viewer with his/her gaze, wherever the viewer stands.

Additionally, remote students are difficult to feel the movements of the other students from lecture video. We conduct a questionnaire survey about the movements of other students in face-to-face lectures. The subjects are 14 graduate and undergraduate students, and all of them have been participating in face-to-face lectures from their childhood. The questions are (1) "Do you obtain any information related to the lecture from the movement or atmosphere of the others?" and (2) "Does the movement or atmosphere of the others affect your motivation or attitude for learning?" The answers for each question consist of four-point Likert scale and open-ended answers.

Table 1 shows the answers of four-point Likert scale questions. We found, from the results, (1) the subjects sometimes obtain information, and (2) they often are affected from the other students. The open-ended answers for the question (1) indicate that the information is mainly related to importance of teacher's explanation, and the trigger movement is note taking. The result of the question (2) shows that the earnestness of the others motivates the student to learn.

From the results, we focus on note-taking behaviour to inform the students the movement of the others.

Table 1 Result of the questionnaire survey about information in a lecture.

\begin{tabular}{ccc}
\hline Question & $(1)$ & $(2)$ \\
\hline 4. Very frequently & 2 & 4 \\
3. Frequently & 6 & 7 \\
2. Rarely & 6 & 3 \\
1. Very Rarely & 0 & 0 \\
\hline Mean & 2.7 & 3.1 \\
S.D. & 0.73 & 0.73 \\
\hline
\end{tabular}




\subsection{Situational Differences in Exchanging Information}

We assume that there are two different situations in a lecture such as "lecturing" and "questions and answers" time, and consider the verbal and nonverbal information between the teacher and the students in each situation. Some lectures include other situations such as "discussing with other students," "collaborating with others," and so on. However, we have not considered such situations as mentioned in Section 2.

Table 2 shows the verbal and nonverbal information, which the teacher and the students receive in lecturing time. In this situation, the teacher one-sidedly talks to the students, and the students listen to the teacher's talk, watch the writing on blackboard and slides, and take notes.

Table 2 Verbal and nonverbal information between the teacher and the students in lecturing time.

\begin{tabular}{lcc}
\hline receiver & verbal & nonverbal \\
\hline teacher & none & $\begin{array}{c}\text { expressions and movements } \\
\text { gaze on the blackboard/slides }\end{array}$ \\
\hline students & $\begin{array}{c}\text { utterances } \\
\text { writing on the blackboard/slides }\end{array}$ & $\begin{array}{c}\text { expressions and gestures of the teacher } \\
\text { movements of the other students }\end{array}$ \\
\hline
\end{tabular}

Table 3 shows the verbal and nonverbal information, which the teacher and the students receive in questions and answers time. In this situation, the teacher and a student exchange their questions and answers. Thus, there are three types of person such as the teacher, a questioner/answerer (one of the students), and other students in the situation.

Table 3 Verbal and nonverbal information among the teacher and the students in questions and answers time.

\begin{tabular}{ccc}
\hline receiver & \multicolumn{1}{c}{ verbal } & nonverbal \\
teacher & $\begin{array}{c}\text { utterances of } \\
\text { the questioner/answerer }\end{array}$ & $\begin{array}{c}\text { expressions and gestures } \\
\text { of the questioner/answerer } \\
\text { and the other students } \\
\text { the questioner's/answerer's gaze }\end{array}$ \\
$\begin{array}{c}\text { questioner/ } \\
\text { answerer }\end{array}$ & $\begin{array}{c}\text { utterances of the teacher } \\
\text { writing on the blackboard/slides }\end{array}$ & $\begin{array}{c}\text { expressions, gestures, } \\
\text { and gaze of the teacher }\end{array}$ \\
\hline other students & $\begin{array}{c}\text { utterances of the teacher } \\
\text { and questioner/answerer } \\
\text { writing on the blackboard/slides }\end{array}$ & $\begin{array}{c}\text { expressions and gestures } \\
\text { of the teacher and } \\
\text { the questioner/answerer }\end{array}$ \\
\hline
\end{tabular}




\section{Overview of Proposed System}

This study proposes a remote lecturing system, which

(1) supports students to synchronously participate in remote lectures anywhere with their computers and narrow network,

(2) distributes lecture video in fixed camera angle to each student's computer,

(3) sends the student's face video to the teacher and the other students,

(4) allows students to clip pictures from the video and take notes on the clipped pictures, and

(5) distributes information to complement nonverbal information.

Figure 2 shows the overview of the proposed system.

Figure 2 The overview of the proposed system.

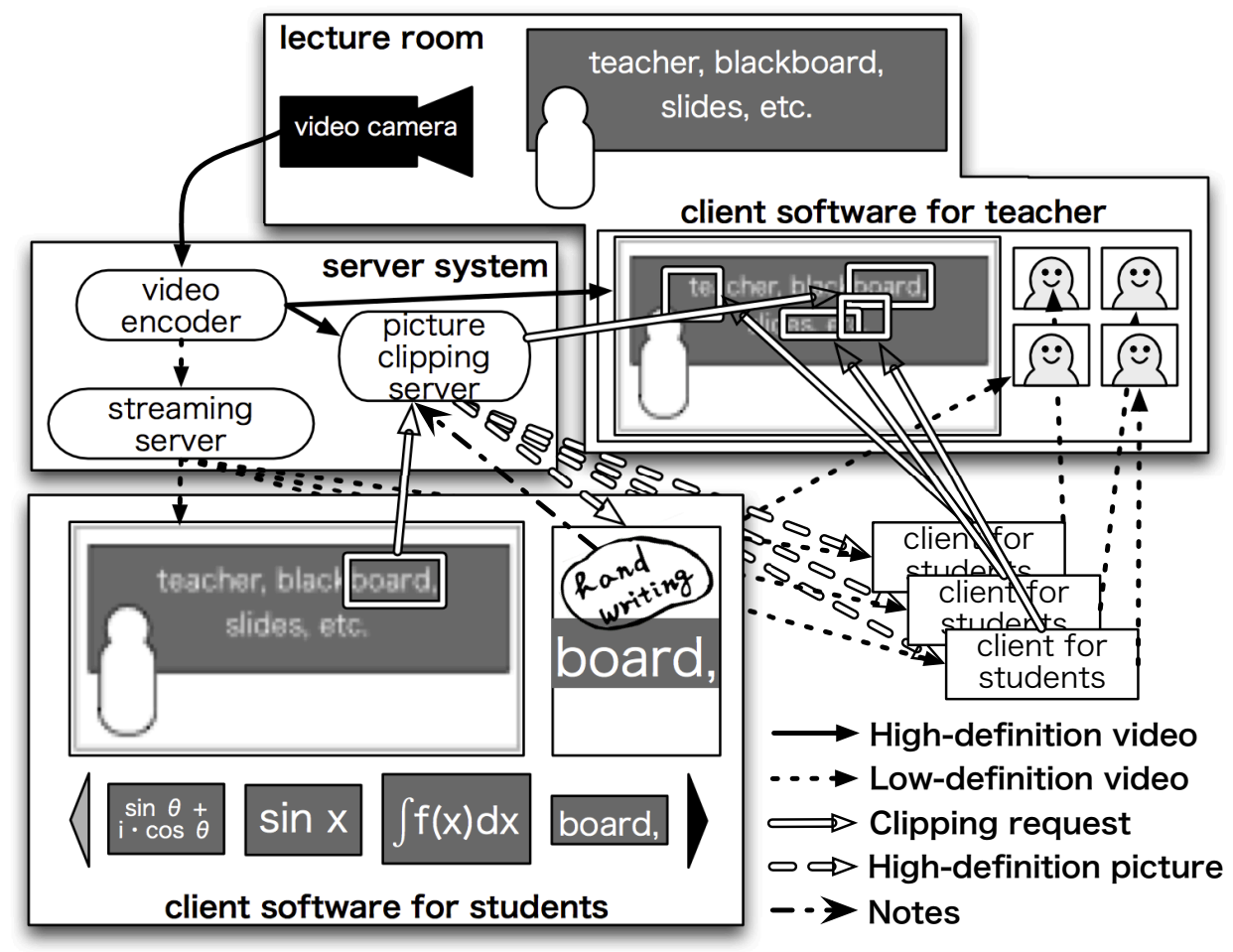

In the lecture room, the teacher, the blackboard, the slides, and so on are recorded with the video camera in fixed angle and high definition $(1920 \times 1080$ pixels $)$. The video encoder receives the video from the camera, and encodes the video into high definition and low definition $(640 \times 360$ pixels or less $)$ videos. The encoder forwards the high definition video to the client software for the teacher and the picture clipping 
server, and low definition video to the streaming server. The streaming server distributes the low definition video to the client software for students.

The client software for students is Flash-based software and works on general web browsers with Flash Player plug-ins. We intend the software to be used by all on-site and remote students and on laptop PCs and tablets with front-facing camera, and sends the student's face video, captured with the camera, to the client software for the teacher.

The students always can see entire the teacher, the blackboard, the slides, and so on in the distributed video; however, they cannot clearly recognise the detail of the writing on the blackboard and slides because the video is in low definition. The software allows the students to clip picture from the video by dragging a region on the video. The client software sends a clipping request, which includes the timestamp of the request and coordinate data of the region, to the picture clipping server when the student drags the region. The clipping server generates a high definition picture from the high definition video and the requested data from the client, and returns the picture to the client. The high definition picture shows the student the detail of the writing on which he/she drags.

Additionally, the students can write characters and freehand drawings on the clipped picture. This feature helps the students to taking notes. The software sends the writing notes to the picture clipping server.

The client software for the teacher is also Flash-based software and shows the high definition video to the teacher. It also presents him/her the regions where each remote student recently clips. The client software for remote students sends a low definition video, which takes the student's face, to the client software for teacher.

\subsection{Client Software for Teacher}

Figure 3 shows the information, which is provided by the client software for the teacher in the lecturing time and the questions and answers time. The software always shows the teacher the regions where the students recently clip. The teacher senses the students' behavior and status, for instance, where they are interested in, and whether they follow the progress of the lecture, from the movement of the presented regions. This information complements the students' gaze on the blackboard or slides. The software also presents the teacher all students' faces in the lecturing time (Fig. 3(a)). This feature helps the teacher to grasp the students' expressions and movements. In the questions and answers time, the software emphasises the video of the questioner's/answerer's face (Fig. 3(b)). This feature helps the teacher to concentrate on his/her expressions and movements.

\subsection{Client Software for Students}

Figure 4 shows the information, which is provided by the client software for the students in the lecturing time and the questions and answers time. The software always shows the students the lecture video (fixed angle, low definition) and the history of clipped pictures. The software also shows the students high definition clipped picture in the lecturing time (Fig. 4(a)). They can write notes and free-hand drawings on the picture to take their notes. The notes are sent to the picture clipping server to collect the movements of the student. When some students are taking notes, the software shows the student those movements to raise their awareness about importance of the teacher's explanation. 
Figure 3 The client software for the teacher.

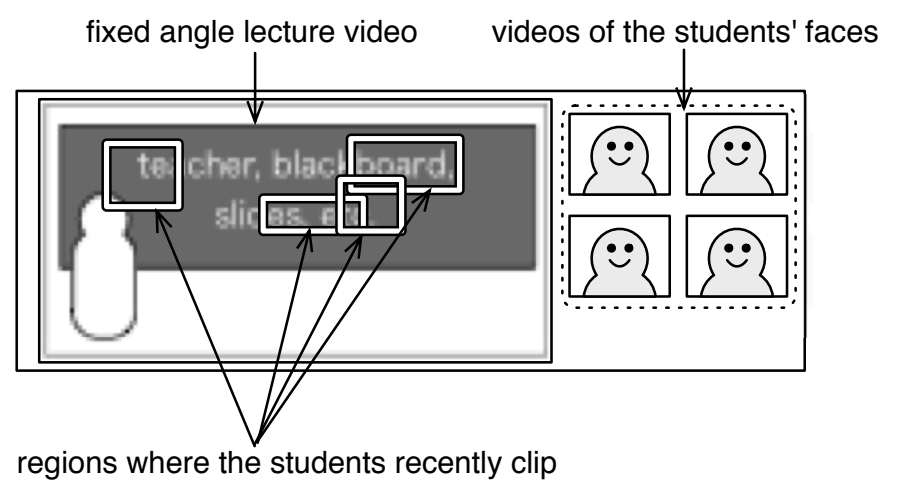

(a) In the lecturing time.

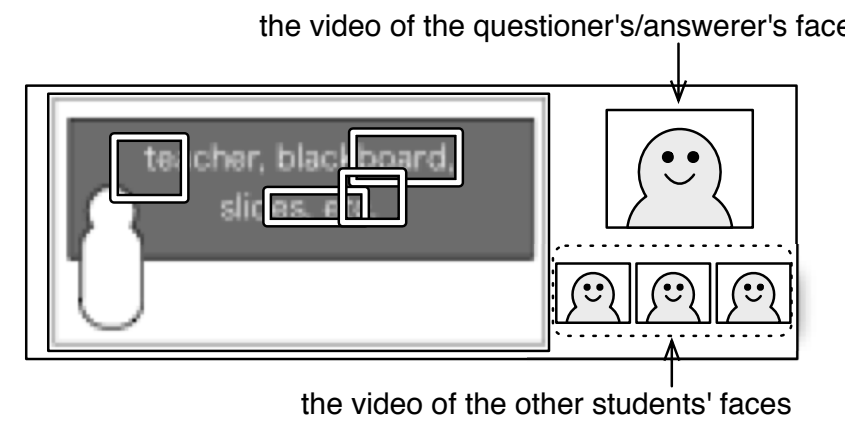

(b) In the questions and answers time.

In the questions and answers time, the software shows the questioner/answerer the video of the teacher's face instead of the clipped picture (Fig. 4(b)). This feature helps the questioner/answerer to feel that the teacher closely observes my behavior. On the other hand, the other students watch the video of the questioner's/answerer's face instead of the clipped picture at that time (Fig. 4(c)). This feature helps them to observe the expressions and movements of the questioner/answerer in addition to the verbal and nonverbal information from the teacher and verbal information from the questioner/answerer.

\section{Preliminary Evaluation}

We conducted a preliminary experiment to evaluate the usability of the proposed system. The subjects of this experiment are nine undergraduates and seven graduate students in a computer science course. The control group consists of four undergraduates and four graduates, and the experimental group consists of five undergraduates and three graduates. 
Figure 4 The client software for the students.

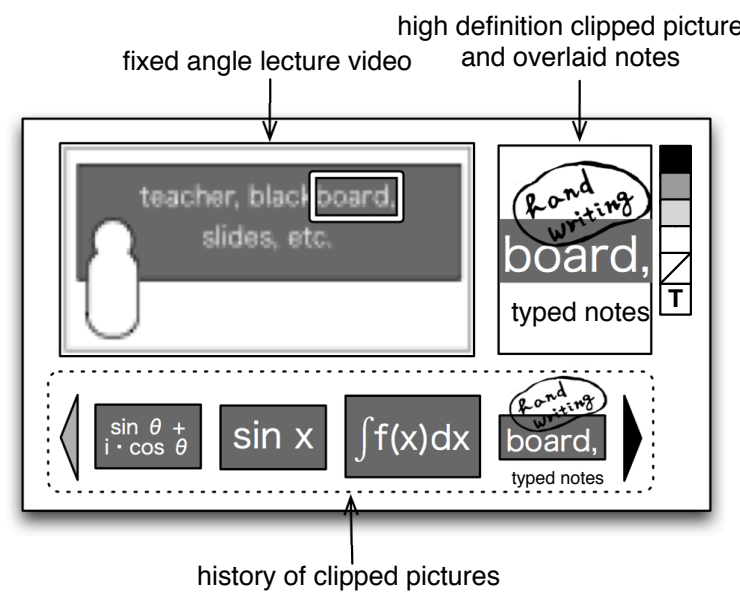

(a) In the lecturing time.

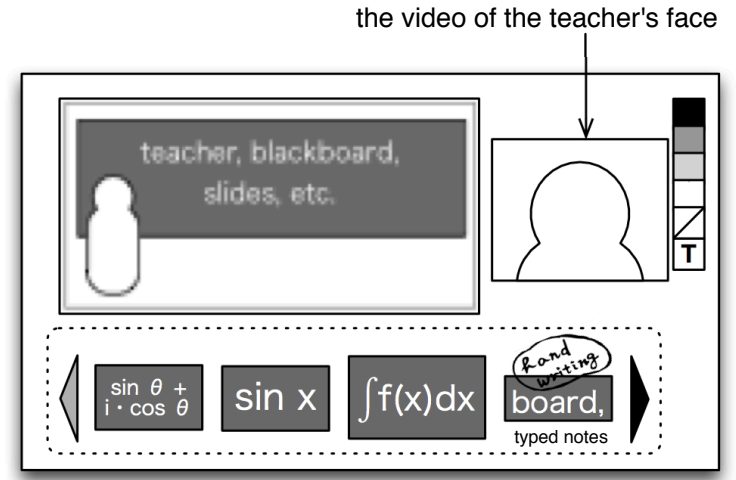

(b) In the questions and answers time (the questioner/answerer).

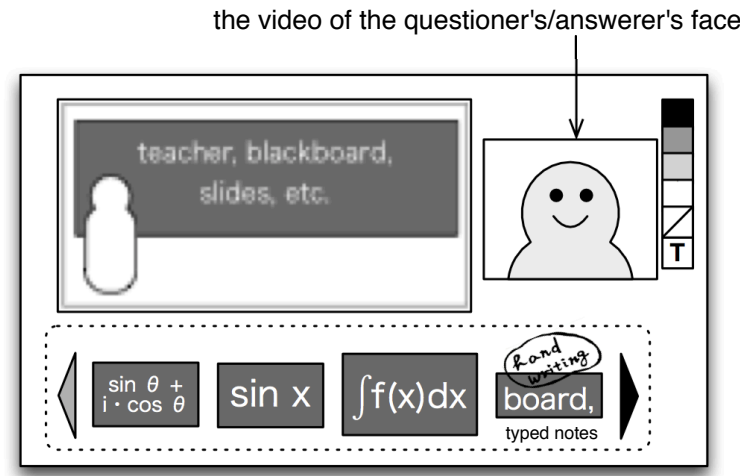

(c) In the questions and answers time (the other students). 


\subsection{Procedure}

At first a teacher gives a lecture in a room, and the control group participates the lecture at other room. The lecture is recorded with a high definition video camera, and the participants simultaneously watches it on a 47 inches display $(1920 \times 1080$ pixels $)$. The teacher does not receive any feedback from the participants.

Next, the teacher gives the same lecture again in the room, and the experimental group participates the lecture at the other room. The lecture is also recorded with a high definition video camera, and each participant simultaneously watch it on his/her PC, which has a 13 inches display $(1280 \times 800$ pixels $)$, with the prototype system. The fixed angle lecture video and the face of the teacher occupy nine inches $(800 \times 600$ pixels $)$ and about four inches $(320 \times 320$ pixels $)$ in the display, respectively. The participants can clip on the lecture video, and the teacher receives the information about clipped regions.

The teacher is skillful and prepares the plan of the lecture enough; thus, the teacher performs much the same in both of the lectures. In these lectures, there is no questions and answers time.

After the lectures, a questionnaire survey is conducted on the system usability of the participants. Additionally, we interview the teacher about the feedback offered by the system.

\subsection{Evaluation of Usability}

We conducted a questionnaire survey on the usability. The questions are about (1) realistic sensation, (2) ease of readability of writing on the blackboard, (3) concentration, (4) note taking, (5) the teacher's facial expression, and (6) fatigue. The answer for each question is in four-points Likert scale.

Table 4 shows the answers of the four-points Likert scale questions. To determine where there are differences in the representative values in the control and experimental groups, the Mann-Whitney $U$ test is performed with the null hypothesis $\left(H_{0}\right)$, which indicates that the "representative values are the same." There are no significant differences in all tests at the five percent level of significance according to the result. This result indicates that the proposed system assists the participants without high definition video on a large display.

The participants in experimental group clip 5.8 pictures in average.

\subsection{Evaluation of Offered Information}

We asked the teacher three questions about information in a lecture and feedback offered by the system. The questions are (1) "Do you change the content of your talk according to students' states in a face-to-face lecture?", (2) "What do you take notice of about students in a face-to-face lecture?", (3) "How do you feel about the offered information (clipped regions) in this experiment?"

The teacher answered these questions as follows:

(1) I sometimes explain the content in detail according to students' reaction.

(2) A nod and the gaze of students are important. 
Table 4 Result of the questionnaire survey about the usability.

(a) Control group

\begin{tabular}{lcccccc}
\hline Question & $(1)$ & $(2)$ & $(3)$ & $(4)$ & $(5)$ & $(6)$ \\
\hline 4. Very good & 2 & 2 & 2 & 2 & 1 & 2 \\
3. Good & 5 & 5 & 4 & 1 & 4 & 3 \\
2. Poor & 1 & 1 & 2 & 5 & 2 & 3 \\
1. Very poor & 0 & 0 & 0 & 0 & 1 & 0 \\
\hline Mean & 3.1 & 3.1 & 3.0 & 2.6 & 2.6 & 2.9 \\
S.D. & 0.64 & 0.64 & 0.76 & 0.92 & 0.92 & 0.83 \\
\hline
\end{tabular}

(b) Experimental group

\begin{tabular}{lcccccc}
\hline Question & $(1)$ & $(2)$ & $(3)$ & $(4)$ & $(5)$ & $(6)$ \\
\hline 4. Very good & 5 & 6 & 3 & 3 & 2 & 1 \\
3. Good & 2 & 2 & 5 & 4 & 3 & 5 \\
2. Poor & 1 & 0 & 0 & 1 & 3 & 2 \\
1. Very poor & 0 & 0 & 0 & 0 & 0 & 0 \\
\hline Mean & 3.5 & 3.8 & 3.4 & 3.3 & 2.9 & 2.9 \\
S.D. & 0.76 & 0.46 & 0.52 & 0.72 & 0.83 & 0.64 \\
\hline
\end{tabular}

(3) In the first lecture, there was no feedback from the students, so it was difficult to set the pace of talk. In the second lecture, I could find where the students watch with the offered information, and it helped to set the pace.

But, when I made mention of previous written figure, the students did not clip the figure again. I missed the students' notice in such situations.

The results of the above-mentioned experiment show that some features of the proposed system are useful. However, there are many features, which have not evaluated in this experiment. Additionally, we find an issue to be improved on showing information for a teacher.

\section{Evaluation Items}

We are improving the prototype system, and have already confirmed that the system can distribute videos and high definition pictures from the lecture room at a university to several students at each home. However, we have to measure the detail of the system performance in actual lectures.

We also plan to evaluate the system from the viewpoints of the adequacy of its functionalities and the effects of offered verbal and nonverbal information. Following items are the evaluation items, which should be confirmed in experiments, of the system we considered.

- At the lecturing time, 
A remote lecturing system offering complementary nonverbal information 13

- the teacher notices the expressions and the movements of each student,

- the students notice the expressions and the gestures of the teacher, and

- the students are aware of the movements of the other students.

- At the questions and answers time,

- the teacher notices the expressions and the gestures of questioner/answerer,

- the questioner/answerer notices the expressions and the gestures of the teacher, and

- the other students notices the interaction between the teacher and the questioner/answerer.

\section{Conclusion}

This paper considers the verbal and nonverbal information in a style of lectures and proposes a remote lecturing system intended to complement nonverbal information. The proposed system has following features.

- The system works with narrow networks by distributing low definition videos.

- Students always can look at where they would like to look in high definition picture by clipping there.

- They also take notes directly on the clipped pictures.

- The system offers the teacher and the students verbal information and complementary nonverbal information depending on the situations of the lecture.

We also conducted a preliminary experiment to evaluate the usability of the system, and considered the items to evaluate the proposed system.

We will operate the system in the actual lectures and evaluate the system according to above-mentioned evaluate items, and improve the system functionalities based on experimental results.

Additionally, we consider the availability to introduce the system into other styles of lectures such as collaborative, grouped, and so on.

\section{References}

[1] Randal Abler, Sean Brennan, and Joel Jackson. High definition video support for natural interaction through distance learning. In Proc. of Frontiers in Education Conference, 2008. FIE 2008. 38th Annual, pages T3C-15-T3C-20, 2008.

[2] I. Elaine Allen and Jeff Seaman. Going the Distance Online Education in the United States, 2011. Babson Survey Research Group and Quahog Research Group, LLC, 2011.

[3] Gudula Naiga Basaza, Natilie B. Milman, and Clayton R. Wright. The challenges of implementing distance education in uganda: A case study. International Review of Research in Open and Distance Learning, 11(2):85-91, 2010. 
[4] Mary Bold, Lillian Chenoweth, and Nirisha K. Garimella. BRICs and clicks. Journal of Asynchronous Learning Networkds, 12(1):5-25, 2008.

[5] Abrar Hasan and Wolfram Laaser. Higher education distance learning in portugal - state of the art and current policy issue. Europian Journal of Open, Distance and E-Learning, 2010(2):1-10, 2010.

[6] Keiji Hirata, Yasunori Harada, Toshihiro Takada, Shigemi Aoyagi, Yoshinari Shirai, Naomi Yamashita, and Junji Yamato. The t-room: Toward the future phone. NTT Technical Review, 4(12):26-33, 2006.

[7] Hisayoshi Kunimune, Yasushi Fuwa, Mizue Kayama, Koichi Miyasaka, and Tadashi Miyosawa. Advancement of synchronous remote lectures with highdefinition videos - differences of learners' impressions by change of camera angles_. JSiSE Research Report, 23(6):64-69, 2009.

[8] Freddy Priem, Renaat De Craemer, Johan Calu, Fran Pedreschi, Thomas Zimmer, Sylvain Saïghi, and Jarmo Lilja. E-learning in science and technology via a common learning platform in a lifelong learning project. European Journal of Open, Distance and E-Learning, 2011(1):1-24, 2011.

[9] Frank Rennie, Sigurbjög Jóhannesdóttir, and Stefania Kristinsdottir. Re-thinking sustainable education systems in iceland: The net-university project. International Review of Research in Open and Distance Learning, 12(4):88-105, 2011.

[10] Richard A Skinner. The challenges of transnational online learning. Journal of Asynchronous Learning Networks, 12(2):83-89, 2008.

[11] The council of the European Union. Council conclusions of 12 May 2009 on a strategic framework for European cooperation in education and training ('ET 2020'). Official Journal of the European Union C 119, 52:2-10, 2009. 